\title{
PATTERNING TECHNIQUES FOR FABRICATION OF SUBMICROMETER STRUCTURES IN PHOTORESIST, III-V SEMICONDUCTORS AND PMMA
}

This paper presents experimental results in the field of planar periodic structures, their fabrication and analysis. We demonstrate techniques and experimental results of fabrication of two-dimensional periodic structures and their preparation in a thin photoresist layer, III-V semiconductors and PMMA - interference lithography using two coherent laser beams, nanoimprint lithography and lithography using nearfield scanning optical microscope.

\section{Introduction}

New applications in photonics and optoelectronics are focused on exploitation of periodic structures known as photonic structures. The photonic structures with submicrometer period enable to change the optical and electrical properties of optic and optoelectronic devices.

Periodic structures can be fabricated by different lithography techniques as holographic lithography [1, 2], electron beam lithography [3], nanoimprint lithography [2, 4, 5] and near-field scanning optical microscope lithography [6-9]. Techniques presented in this paper allow maskless definition of surface microstructures.

One of the simplest ways how to obtain microstructures with two-dimensional geometry (2D) is holographic lithography based on a double-exposure process using two-beam interference optical field $[1,5,6]$. In this way, the holographic lithography produces high-resolution periodic structures in a large area with low costs. These periodic structures patterned in a thin photoresist layer can be employed as a mask for the patterning of III-V semiconductor surfaces, which may be attractive for optoelectronic and photonic applications [10]. However, due to the diffraction limit, it is very difficult for the holographic lithography to reach the subwavelength resolution. The subwavelength resolution can be achieved by submicron-diameter optical fiber, where the metal coated fiber tip is used as a subwavelength fiber probe for a local exposure [6]. A combination of such a fiber probe with nanopositioning system can be used as the near field scanning optical microscope lithography with high exposure resolution.

Unlike the above optical forms of patterning that employ radiation to delineate a pattern in a resist, non-radiation based patterning uses a mechanical mold that shapes a material into features.
One of these techniques is nanoimprint lithofraphy (NIL). As the NIL's working principle is fundamentally different from radiationbased lithography, it has many advantages over conventional lithography, as high pattern transfer fidelity, larger area and high throughput $[4,5]$. NIL does not use any energetic beams; therefore its resolution is not limited by the effects of wave diffraction, scattering and interference in a resist and backscattering from a substrate. Moreover, they can directly pattern functional materials to reduce fabrication steps.

In this work we present experimental results from holographic lithography of two beam interference method and near field scanning optical microscope lithography as effective optical techniques for the preparation of 2D periodic structures in a thin photoresist layer as well as on GaAs surfaces. Non-optical possibility of structure patterning was examined in PMMA material using nanoimprint lithography.

\section{Two-Dimensional Structures Patterned by Interference Lithography}

Holographic lithography using two beam geometry allows to design one and two dimensional periodic structures by a simple theoretical approach of interference of two optical plane waves. The intensity distribution of the interference pattern of two optical plane waves with the same intensity in the $x y$ plane can be expressed as [1]

$$
I_{\alpha}=4 I_{0} \cos ^{2}[k \sin \theta(x \cos \alpha+y \sin \alpha)],
$$

where $I_{0}$ is the intensity of the interfering beams, $k$ is the wavenumber, $\theta$ is the semiangle between two interfering beams and the

\footnotetext{
* D. Pudis ${ }^{1}$, I. Kubicova ${ }^{1}$, L. Suslik ${ }^{1}$, J. Skriniarova ${ }^{2}$, I. Martincek ${ }^{1}$, I. Novotny ${ }^{2}$

${ }^{1}$ Dept. of Physics, University of Zilina, Slovakia

${ }^{2}$ Dept. of Microelectronics, Slovak University of Technology, Bratislava, Slovakia, E mail: pudis@fyzika.uniza.sk
} 
angle $\alpha$ represents the sample orientation in the $x y$ plane (Fig. 1). The optical field periodicity $\Lambda$ is then determined by

$$
\Lambda=\frac{\lambda}{2 \sin \theta},
$$

where $\lambda$ is the wavelength of the interfering beams.

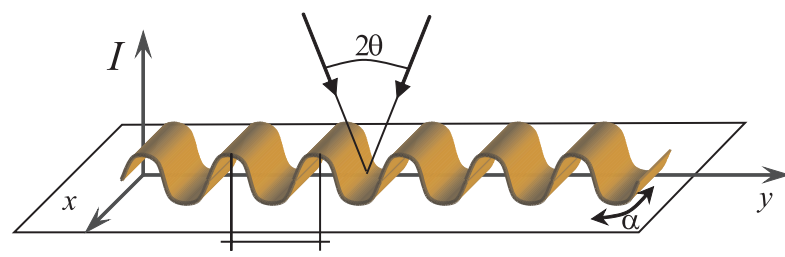

$\Lambda$

Fig. 1 In-plane intensity distribution of two interfering beams containing angle $2 \theta$ for defined sample rotation a according eq. (1)

The schematic in-plane distribution shown in Fig. 1 documents only 1D field distribution. The multiexposure process is necessary for the preparation of $2 \mathrm{D}$ periodic structures. In case of double exposure at different angles of sample orientation $\alpha$, the exposure dose is accumulated and the total exposure is the sum of the partial exposures $I_{t o t}(x, y)=I_{1}+I_{2}$ what finally forms 2D optical field distribution.

\subsection{Experiment}

Periodic structures can be prepared on different metal and semiconductor surfaces by interference lithography. In this experiment the interference optical field is formed by the interference of two coherent beams of the Argon ion laser operating at $488 \mathrm{~nm}$. A positive photoresists AZ 5214E layer is used as a mask [11]. The 10x expander was used for improving the exposure homogeneity in the exposed area with a diameter of $5 \mathrm{~mm}$ which resulted in the exposure intensity of $25 \mathrm{~mW} / \mathrm{cm}^{2}$ both laser beams.

For the patterning of GaAs samples, n-doped GaAs substrates were covered with $0.7 \mu \mathrm{m}$ thick photoresist film as a mask. The photoresist film was spin-coated with post-baking at $103{ }^{\circ} \mathrm{C}$ for 50 seconds. After the exposure and photoresist development, the samples were etched by Reactive ion etching (RIE) in $\mathrm{CCl} 4 / \mathrm{He}$ based plasma in ROTH \& RAU MICROSYS 350 machine [10]. Only a weak absorption is documented for this type of employed photoresist in the region of the Ar laser wavelength, which effects insufficient polymerization of the photoresist at short exposition times. Then the sample exposure was realized at exposition time in the range of 10-12 min. Finally the structure quality was examined by an atomic force microscope (AFM).

\subsection{Results and discussion}

2D photonic structures with square symmetry were prepared on GaAs surface using a double exposure process with sample in- plane rotation between the exposures at angle $\alpha=90^{\circ}$. The period of $2 \mathrm{D}$ photonic structure was set in the experiment to be $\Lambda=2.0 \mu \mathrm{m}$.

The distribution of 2D optical field in a double exposure process typically can be described by a sinus function in $x y$ plane [1]. The sinusoidal shape was formed into the exposed photoresist layer with the depth of approximately $600-700 \mathrm{~nm}$ in the developing process with duration of 25-30 s [11]. Additional developing starts a formation of the open regions in the photoresist mask (30-40 s). The additional developing process ( $>40 \mathrm{~s})$ enlarges the open regions. Photoresist masks prepared in this way with different shaped open regions were used for the formation of $2 \mathrm{D}$ structures onto GaAs surface.
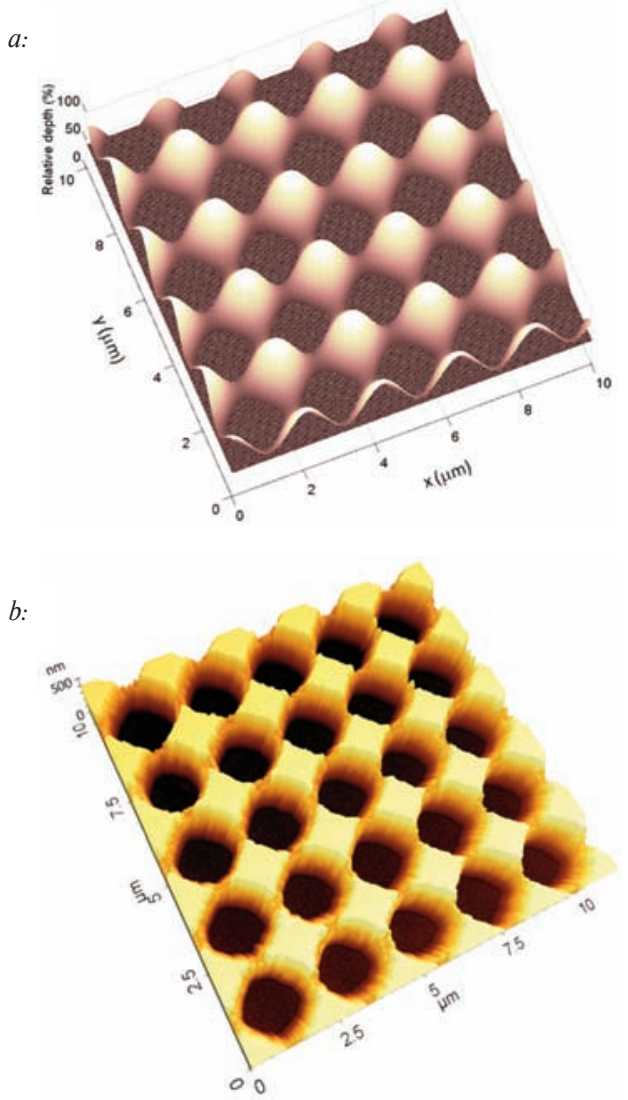

Fig. 2 a) Simulation of the open regions (dark area) in the photoresist mask, b) AFM image of prepared $2 D$ photonic structure in the GaAs substrate

The following RIE process preserves the shape of the photoresist mask in GaAs substrate. Fig. 2a demonstrates a simulation of photoresist patterning on the GaAs surface after developing. AFM image (Fig. 2b) shows the real etched structure in GaAs substrate with the removed regions. These regions correspond to the nearly 
square shape of the photoresist mask (Fig. 2a). By etching the deep structures (app. $500 \mathrm{~nm}$ ), the final shape of structure reflects also the photoresist mask thickness. It is evident on the barriers overetching, where the thickness of photoresist mask is the lowest. This method allows the formation of open regions of different size depending on the developing time, as well as on the time of RIE process and experiment geometry [10, 11].

\section{Nanoimprint in PMMA}

Nanoimprint lithography has two basic steps as shown in Fig. 3. The first is the imprint step in which a mold with nanostructures on its surface is pressed into a thin resist cast on a substrate, followed by the removal of the mold. This step duplicates the nanostructures on the mold in the thin resist layer. The second step is the pattern transfer where an anisotropic etching process, such as RIE, is used to remove the residual resist in the compressed area. This step transfers the thickness contrast pattern into the entire resist [5].

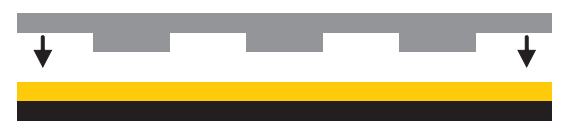

mold

resist

substrate

a)

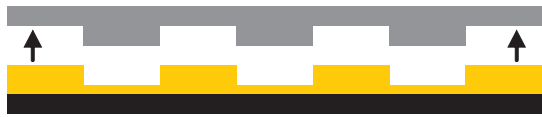

b)

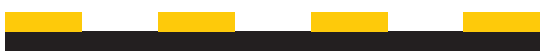

c)

Fig. 3 Schematic of nanoimprint lithography process: a) imprinting by pressing the mold, b) mold removal, c) residual resist removal by anisotropic etching process [5]

\subsection{Experiment}

In our experiment, GaAs was used as the mold material. The mold was patterned by two-beam interference holographic lithography shown in Fig. 2b. The period of the prepared mold structure was $2.0 \mu \mathrm{m}$ and the intrusion of about $550 \mathrm{~nm}$. Because of the small pressure shrinkage coefficient, the acetone solution of polymethyl methacrylate (PMMA) was used as the resist. The PMMA layer thickness was kept thicker than the mold intrusion to prevent the mold from contacting the substrate. The PMMA structure quality was examined by AFM after the process.

\subsection{Results and discussion}

The period of the imprinted PMMA structure is $2.0 \mu \mathrm{m}$ and the depth is of about $250 \mathrm{~nm}$ as shown in AFM image (Fig. 4).

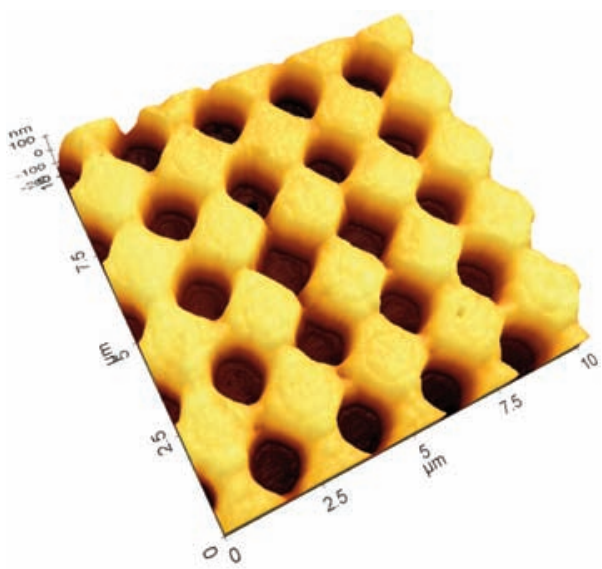

Fig. 4 AFM image of $2 D$ periodic structure prepared in thin PMMA layer

When comparing the imprinted PMMA with the mold characteristics (Fig. 2b), the structure period is left unchanged, but the depth is lower than the mold intrusion. The fact is caused by the presence of air during imprinting. However, nanoimprint using GaAs mold may be a promising technique achieving better than submicrometer resolution if applied in vacuum.

\section{Nanopatterning Using NSOM Lithography}

Among different types of existing methods for fabrication of nanostructures, near-field scanning optical microscope (NSOM) lithography is one of the favored techniques. The patterning of nanostructures is done through a direct writing process which is performed by the optical near-field produced at the tip of a fiber probe. Therefore, the fabrication of nanostructures in a size below the diffraction limit of the light source that poses the ultimate resolution limit in conventional optical lithography is possible [6]. Also there is no need for mask preparation as it is in photolithography, and the patterning can be performed in air. On the other hand, as the interaction between the optical near-field and the sample surface is a photochemical process, conventional materials used in far-field optical lithography, for example photoresists, can be used $[6,7,12]$.

\subsection{Experiment}

We focused on experimental investigation of NSOM lithography for the preparation of structures using a conventional nanopositioning stage and a fiber probe. A schematic diagram of the experimental arrangement is shown in Fig. 5. A $473 \mathrm{~nm}$ continuous wave DPSS laser was used as the light source. The laser beam was focused into conventional optical fiber, which was coupled to the fiber probe. Even though interesting results can be obtained using the contact mode $[6,12]$, we arranged non-contact mode of NSOM lithography. In the non-contact mode the fiber was fixed on the $3 \mathrm{D}$ nanopositioning piezosystem to realize the $3 \mathrm{D}$ movement of 
the fiber probe over the sample. Two different types of fiber probe were used, a conventional optical fiber of $10 \mu \mathrm{m}$ core diameter and the metal-coated fiber tip with a submicrometer aperture on the output side.

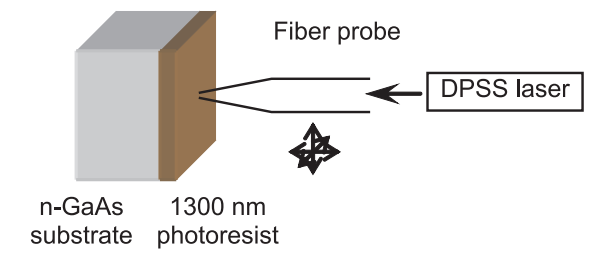

Fig. 5 Experimental arrangement for NSOM lithography

The positive photoresist AZ 5214E was used for patterning. For the sample preparation, the $1300 \mathrm{~nm}$ thick photoresist film was spin-coated on a GaAs substrate. The patterning of the photoresist was carried out by moving the fiber probe over the sample while the laser light was irradiated through the fiber probe aperture. After exposure, the sample was developed in AZ 400K developer for $30 \mathrm{~s}$, rinsed in DI water. The structure quality was examined using an optical microscope. Dektak 150 Stylus Profiler using Vision 3D advanced analysis software was used to determine topography of dots prepared by metal-coated fiber tip.

\subsection{Results and discussion}

The patterning of dots as well as lines was tested using the conventional optical fiber of $10 \mu \mathrm{m}$ core diameter. This fiber probe was placed approximately $10 \mu \mathrm{m}$ from the sample surface. Different structures prepared by NSOM lithography are shown in Fig. 6. The lattice in Fig. $6 \mathrm{~b}$ was created by exposure of 5 horizontal and 5 vertical lines with $50 \mu \mathrm{m}$ pitch step. When using the $10 \mu \mathrm{m}$ core diameter optical fiber, the line width of $12 \mu \mathrm{m}$ was achieved. This line widening is caused by a divergence of the irradiated optical field at the end of the fiber probe.

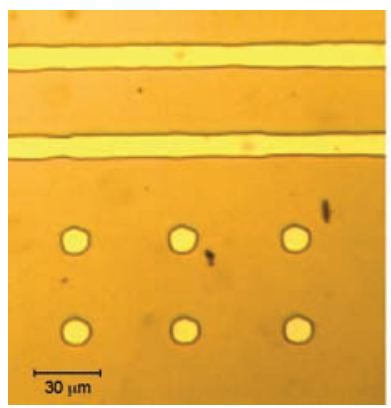

a)

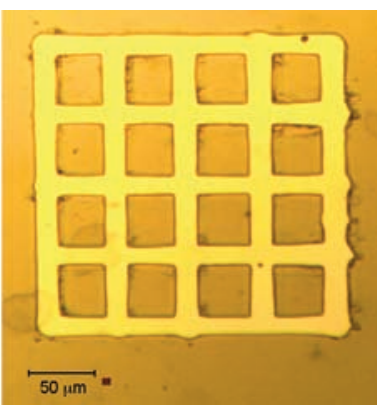

b)
Fig. 6 a) Lines and dots; b) lattice exposed by optical fiber

To shrink the line width of the structure and to improve the technique resolution, the metal coated fiber tip with a smaller aperture was used. The results using the metal coated fiber tip demon- strate the resolution improvement to be lower than $3 \mu \mathrm{m}$. An array of 3 by 3 dots was exposed as shown in Fig. 7. The exposure time was set to one, two and three seconds for each dot in the $1^{\text {st }}$, $2^{\text {nd }}$ and $3^{\text {rd }}$ line, respectively.

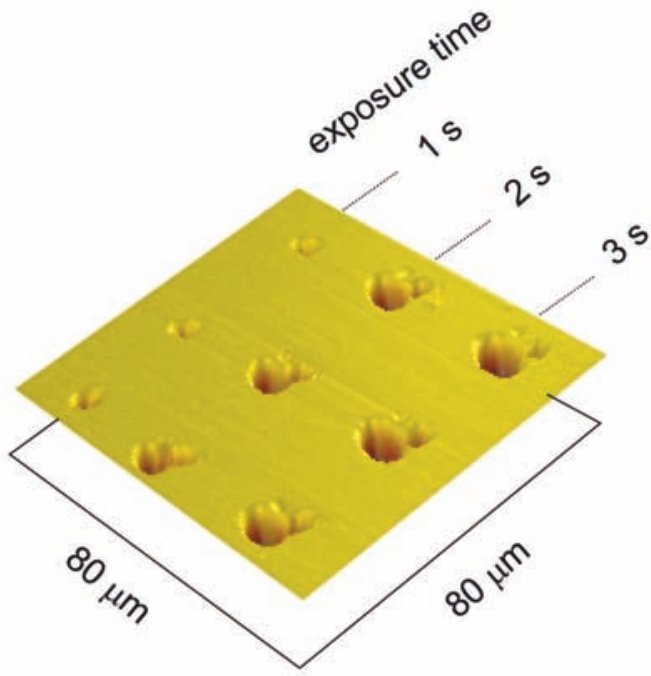

Fig. 7 Dektak image of dots exposed by NSOM lithography using the metal coated fiber tip

Dektak image shown in Fig. 7 documents a dependence of dots diameter on the exposure time. The longer exposure time forms the dots of a higher diameter. The smallest dots of a diameter lower than $3 \mu \mathrm{m}$ were achieved at $1 \mathrm{~s}$ exposure time. The diameter of prepared open regions is then function of the exposure time, because of the gaussian shape of the optical field of the metal coated fiber tip. Therefore, by keeping shorter exposition times the submicrometer resolution is expected. On the other side, the exposed area reflects a non-homogeneity of the prepared fiber tip. Then, the final pattern slightly differs from circular shape as it is documented in Fig. 7.

\section{Conclusion}

The subject of the paper was the investigation of techniques for the fabrication of planar periodic structures and their possibilities to fabricate periodic structures in the photoresist material, GaAs and PMMA surfaces. The two beam interference method was presented as an effective tool for the fabrication for $2 \mathrm{D}$ periodic structures with a small periodicity by using the standard photoresist. The shape and period of the formed structure can be simply adjusted by experiment geometry. This method was successfully examined for the preparation of 2D periodic structures with a period of $2 \mu \mathrm{m}$ in thin photoresist film deposited on the GaAs surface.

The patterned 2D periodic structure on GaAs substrate was used as a mold for the nanoimprint technique. The inverse structures were successfully imprinted in PMMA layer. 
The fabrication of microstructures was examined by NSOM lithography as well. The planar pattern consisting of dots and lines was examined by NSOM lithography using a standard optical fiber probe as well as using a metal coated fiber tip, where the resolution better than $3 \mu \mathrm{m}$ was achieved.

The variability of presented techniques opens wide possibilities to form submicrometer periodic structures on different surfaces of organic and semiconductor materials, which favors these lithography techniques for applications in the region of photonic and electronic devices.

\section{Acknowledgement}

This work was partly supported by the Slovak National Grant Agency No. VEGA 1/0868/08, 1/0220/09, 1/0689/09 and $1 / 0683 / 10$. The authors wish to thank for the support to the R\&D operational program Centre of excellence of power electronics systems and materials for their components. The project is funded by the European Community, ERDF - European regional development fund.

\section{References}

[1] LAI, N.D., LIANG, W.P., LIN, J.H., HSU, C.C., LING, CH. H.: Opt. Express 13, 2005, pp. 9605-9611.

[2] KIM, S. H., LEE, K.D., KIM, J.Y., KWON, M.K., PARK, S.J.: Nanotechnology 18, 2007, p. 055306.

[3] VITTORIO, M. DE, TODARO, M.T., STOMEO, T., CINGOLANI, R., COJOC, D., FABRIZIO, E. DI: Microelectron. Eng., 2004, pp. 73-74.

[4] MURPHY, P.F., MORTON, K.J., FU, Z., CHOU, S.Y.: Appl. Phys. Lett. 90, 2007, p. 203115.

[5] CHOU, S.Y., KRAUSS, P.R., RENSTROM, P.J.: J. Vac. Sci. Technol. B 14, pp. 4129-4133.

[6] WEGSCHEIDER, S., KIRSCH, A., MLYNEK, J., KRAUSCH, G.: Thin solid films 264, 1995, pp. 264-267.

[7] KWON, S., CHANG, W., JEONG, S.: Ultramicroscopy 105, 2005, pp. 316-323.

[8] DRYAKHLUSHIN, V.F., KLIMOV, A. YU., ROGOV, V.V., VOSTOKOV, N.V.: Appl. Surf. Sci. 248, 2005, pp. 200-203.

[9] LIN, Z.CH., YANG, CH.B.: Scanning 28, 2006, pp. 32-41.

[10] PUDIS, D., SKRINIAROVÁ, J., MARTINCEK, I., KOVAC, J. JR., TARJANYI, N., HASCIK, S.: J. Electr. Eng. 60, 2009, pp. 166-169.

[11] SKRINIAROVA, J., PUDIS, D., MARTINCEK, I., KOVAC, J., TARJANYI, N., VESELY, M., TUREK, I.: Microelectron. J. 38, 2007, pp. 746-749.

[12] TIAN, F., YANG, G., BAI, J., XU, J., HOU, CH., LIANG, Y., WANG, K.: Opt. express 17, 2009, pp. 19960-19968. 